\title{
Ischaemic preconditioning does not protect the heart in obese and lean animal models of Type 2 diabetes
}

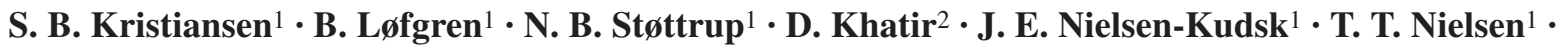 \\ H. E. Bøtker ${ }^{1}$ A. Flyvbjerg ${ }^{2}$ \\ ${ }^{1}$ Department of Cardiology, Skejby Sygehus, Aarhus University Hospital, Aarhus N, Denmark \\ ${ }^{2}$ Medical Research Laboratory / Medical Department M, Aarhus Sygehus, Aarhus University Hospital, Aarhus, Denmark
}

\begin{abstract}
Aims/hypothesis. The prevalence of Type 2 diabetes mellitus is increasing worldwide with obese diabetic patients constituting the majority of this population. Type 2 diabetes is associated with increased morbidity and mortality after acute myocardial infarction. Previous experimental studies of ischaemia-reperfusion tolerance in diabetes have only been performed in animal models of Type 1 diabetes mellitus, yielding conflicting data. The aim of the present study was to characterise and compare the tolerance to ischaemia and effects of ischaemic preconditioning (IPC) in hearts from obese Zucker diabetic fatty (ZDF) and lean Goto-Kakizaki (GK) Type 2 diabetic rats, using nonobese Zucker and Wistar rats as respective controls. Methods. The two rat strains were divided into 8 groups. The ZDF study ( $n=47)$ consisted of: Control -IPC, Control +IPC, ZDF -IPC and ZDF +IPC. The GK study $(n=38)$ consisted of: Control - IPC, Control + IPC, GK -IPC and GK +IPC. Hearts, which were studied in a Langendorff preparation perfused with Krebs-Henseleit buffer, were subjected or not to IPC
\end{abstract}

(+IPC, -IPC) before 50 minutes of regional ischaemia and 120 minutes reperfusion.

Results. Ischaemic reperfusion injury was smaller in obese $(p<0.05)$ and lean $(p<0.05)$ Type 2 diabetic animals than in their respective control animals. IPC reduced ischaemic reperfusion injury during reperfusion in non-diabetic control rats $(p<0.01)$, but failed to protect hearts from both diabetic animal models. Postischaemic haemodynamic recovery was impaired in the $\mathrm{ZDF}$ rats compared to both control and GK rats $(p<0.05)$.

Conclusions/interpretation. Ischaemic preconditioning does not protect hearts from obese or lean Type 2 diabetic animals. However, the susceptibility of the Type 2 diabetic myocardium to ischaemic damage is lower than in non-diabetic hearts. The method described here could be used as a tool to study the pathogenesis of increased cardiovascular morbidity and mortality in Type 2 diabetes.

Keywords Diabetes mellitus - Ischaemia - Myocardial infarction $\cdot$ Obesity $\cdot$ Reperfusion
Received: 17 March 2004 / Accepted: 10 June 2004

Published online: 7 October 2004

C) Springer-Verlag 2004

\author{
S. B. Kristiansen ( $)$ \\ Department of Cardiology, Skejby Sygehus, \\ Aarhus University Hospital, Brendstrupgaardsvej 100, \\ 8200 Aarhus N, Denmark \\ E-mail: sbk@iekf.au.dk \\ Tel.: +45-89495517, Fax: +45-89496009
}

Abbreviations: AAR, area at risk - ALV, area of left ventricle · GK, Goto-Kakizaki · IPC, ischaemic preconditioning · IS, area of infarction - LVDP, left ventricular developed pressure ·

LVP, left ventricular pressure - RPP, rate pressure product .

ZDF, Zucker diabetic fatty

\section{Introduction}

The prevalence of Type 2 diabetes is increasing worldwide and now runs at $13 \%$ in people aged 60 years and older [1,2]. Obese Type 2 diabetic patients constitute more than $85 \%$ of the Type 2 diabetes population, with lean patients making up less than $15 \%$ [3]. Type 2 diabetes is associated with an increased incidence of congestive heart failure and death after acute myocardial infarction. Although patients with Type 2 diabetes have more severe and diffuse coronary artery disease than non-diabetic patients, reduced myocardial tolerance to ischaemia has also been considered responsible for the 
increased morbidity of ischaemic heart disease in diabetes [2]. However, knowledge about the tolerance to ischaemia-reperfusion and the effect of ischaemic preconditioning (IPC) in the diabetic heart is conflicting and only based on findings in Type 1 diabetes [4].

It is a well-known phenomenon that Type 2 diabetes is dominated by hyperinsulinaemia, hyperglycaemia and dyslipidaemia, while Type 1 diabetes is characterised by insulinopenia, hyperglycaemia and a largely unaffected lipid profile. Furthermore, the onset of Type 1 diabetes is acute, unlike that of Type 2 diabetes, which is characterised by a period of insulin resistance, hyperinsulinaemia and euglycaemia preceding the onset of hyperglycaemia. The differences in metabolic aberration and the onset of disease in the two types of diabetes may affect cardiac responsiveness to ischaemia-reperfusion and IPC. Importantly, tolerance to ischaemia-reperfusion and the ability of IPC to protect the heart prior to ischaemia have not been studied previously in experimental models of Type 2 diabetes. The Zucker diabetic fatty (ZDF) rat and the Goto-Kakizaki (GK) rat are well characterised models of obese and lean Type 2 diabetes respectively $[5,6,7,8]$. The aim of the present study was to investigate the response to ischaemia and IPC in these two animal models of the disease.

\section{Materials and methods}

Animals and study design. The rats were handled according to Danish national guidelines and the guidelines of the American Heart Association for animal research. Animals were fed an ordinary laboratory chow diet. Two different models of Type 2 diabetes mellitus and their respective controls were used. Zucker diabetic fatty (ZDF) rats (homozygote $[f a / f a]$ ), ZDF controls (heterozygote [ $\mathrm{fal}-]$ ) (Charles River Laboratories, Wilmington, Mass., USA), Goto-Kakizaki (GK) rats and GK controls (Wistar) (Taconic M\&B, Eiby, Denmark) were purchased at 6 to 8 weeks of age and were studied at the age of 16 weeks. The animals were divided into 8 groups. The ZDF study ( $n=47)$ consisted of: (i) Control without IPC (heterozygote [fa/-], $n=11$ ); (ii) Control with IPC (heterozygote [ $\mathrm{fa} /-\mathrm{f}$, $n=12$ ); (iii) ZDF without IPC (homozygote [fa/fa], $n=12$ ); and (iv) ZDF with IPC (homozygote $[f a / f a], n=12$ ). The GK study $(n=38)$ consisted of (i) Control without IPC (Wistar, $n=10$ ); (ii) Control with IPC (Wistar, $n=10$ ); (iii) GK without IPC $(n=9)$; and (iv) GK with IPC $(n=9)$.

Assessment of biochemical parameters and in vivo blood pressure. Serum levels of glucose, insulin, cholesterol, triglycerides and NEFA were measured after 8 hours of fasting. In a subset of animals blood pressure was measured by tail plethysmography.

Isolated perfused heart. Rats were anaesthetised with midazolam (Roche, Basel, Switzerland, $0.25 \mathrm{mg} / \mathrm{kg}$ bodyweight intramuscular) and fluanisone (Janssen-Cilag, Beerse, Belgium, $0.5 \mathrm{mg} / \mathrm{kg}$ bodyweight intramuscular). A tracheotomy was made and the rat was connected to a ventilator (Zoovent, Newport Pagnell, UK). Through a common laparotomy and thoracotomy the heart was dissected from surrounding structures. A bolus of $1.000 \mathrm{IE} / \mathrm{kg}$ heparin (Leo Pharma, Copenhagen, Denmark) was given through the femoral vein. The heart was cannulated in situ, mounted in a Langendorff apparatus and perfused retrogradely with Krebs-Henseleit solution $(\mathrm{NaCl}$ $118.5 \mathrm{mmol} / 1, \mathrm{KCl} 4.7 \mathrm{mmol} / \mathrm{l}, \mathrm{NaHCO}_{3} 25.0 \mathrm{mmol} / \mathrm{l}$, glucose monohydrate $11.1 \mathrm{mmol} / \mathrm{l}, \mathrm{MgSO}_{4} \cdot 7 \mathrm{H}_{2} \mathrm{O} 1.2 \mathrm{mmol} / \mathrm{l}, \mathrm{CaCl}_{2}$ $2.4 \mathrm{mmol} / \mathrm{l}$ and $\mathrm{KH}_{2} \mathrm{PO}_{4} 1.2 \mathrm{mmol} / \mathrm{l}$ ) at a pressure of $100 \mathrm{~cm}$ $\mathrm{H}_{2} \mathrm{O}$. The perfusion solution was oxygenated with a mixture of $95 \% \mathrm{O}_{2}$ and $5 \% \mathrm{CO}_{2}$ and kept at $37{ }^{\circ} \mathrm{C}$. A snare was placed around the left main coronary artery $2 \mathrm{~mm}$ from its origin. The hearts were allowed to stabilise for $40 \mathrm{~min}$, subjected to regional ischaemia for $50 \mathrm{~min}$ and further studied during $120 \mathrm{~min}$ of reperfusion. Animals subjected to IPC were exposed to 4 cycles of $2 \mathrm{~min}$ ischaemia and $3 \mathrm{~min}$ reperfusion during the last $20 \mathrm{~min}$ of the stabilisation period. The sustained 50 -minutes ischaemic period was induced by tightening the snare around the left coronary artery.

Evaluation of left ventricular function. A latex balloon (Hugo Sachs Elektronik, March-Hugstetten, Germany) was placed in the left ventricle through an incision in the left atrium and kept in place by the mitral valve. The volume in the balloon was adjusted to obtain an end diastolic pressure of $7 \mathrm{~cm} \mathrm{H}_{2} \mathrm{O}$. A pressure transducer (Baxter Cardiovascular Group, Irvine, Calif., USA) was connected to the latex balloon to record left ventricular pressure (LVP). The analogue signal from the transducer was converted (AD-converter, MP100 system; BioPAC Systems, Goleta, Calif., USA) and stored on a PC. Coronary flow was monitored continuously by a flowmeter (Transonic, Maastricht, the Netherlands).

Assessment of myocardial infarction. While the heart was still in the Langendorff preparation, the area at risk (AAR) was defined by ligating the left coronary artery at the site of the occlusion and infusing $3 \mathrm{ml}$ of $1 \%$ solution of fluorescent microspheres (Duke Scientific, Palo Alto, Calif., USA), leaving the AAR unstained. Hearts were frozen for 15 minutes at $-20{ }^{\circ} \mathrm{C}$ and sliced into $1.5-\mathrm{mm}$ slices. At $37^{\circ} \mathrm{C}$ and $\mathrm{pH}=7.4$ the slices were immersed in 1\% 2,3,5-triphenyltetrazoliumchloride (Sigma, St.Louis, Mo., USA) for 10 minutes to delineate areas of infarction. The hearts were then stored overnight in $2 \mathrm{ml}$ of Lillie's solution (4\% formaldehyde buffer; VWR International, Albertslund, Denmark) to enhance the colour contrasts. The following day each slice was weighed and photographed with a digital camera (Nikon Coolpix 5700, Nikon, Japan) in wolfram and ultraviolet light $(\lambda=530 \mathrm{~nm})$. The AAR, area of left ventricle (ALV) and area of infarction (IS) were assessed using image analysis software (Analysis [Build 776]; Soft Imaging Systems, Munster, Germany). The IS : AAR and AAR : ALV ratios were then calculated. Measurements were weighted with the weight of each individual slice. All analyses were blinded.

Statistics and calculations. All values are expressed as the mean \pm SEM. Left ventricular developed pressure (LVDP) was calculated as left ventricular systolic pressure-left ventricular diastolic pressure. Rate-pressure-product (RPP) was calculated as LVDP $\times$ heart rate. Groups were compared using 2-way ANOVA with repeated measures. IS : AAR was compared by one-way ANOVA and coronary flow by 2-way ANOVA repeated measures. SPSS 10 (SPSS, Chicago, Ill., USA) was used for statistical calculations and a $p$ value of less than 0.05 was considered statistically significant.

\section{Results}

Biochemical parameters and blood pressure. The obese ZDF rats were characterised by hyperglycaemia, hyperinsulinaemia, hypercholesterolaemia and 
Table 1. Clinical and metabolic parameters in Zucker diabetic fatty (ZDF) rats and Goto-Kakizaki (GK) rats at termination of the study

\begin{tabular}{|c|c|c|c|c|}
\hline & \multicolumn{2}{|c|}{ Obese Type 2 diabetes (ZDF) } & \multicolumn{2}{|c|}{ Lean Type 2 diabetes (GK) } \\
\hline & Control group & Diabetic group & Control group & Diabetic group \\
\hline Fasting glc (mmol/l) & $5.7 \pm 0.1$ & $20.5 \pm 0.8 * * *$ & $4.8 \pm 0.1$ & $8.8 \pm 0.4 * * *$ \\
\hline P-insulin $(\mu g / 1)$ & $0.42 \pm 0.02$ & $3.90 \pm 0.4 * * *$ & $1.60 \pm 0.11$ & $2.11 \pm 0.10$ \\
\hline P-NEFA (mmol/l) & $0.53 \pm 0.01$ & $0.81 \pm 0.04 * * *$ & $0.83 \pm 0.04$ & $0.64 \pm 0.02 * * *$ \\
\hline MAP (mm Hg) & $127 \pm 6$ & $148 \pm 5^{* *}$ & $117 \pm 4$ & $120 \pm 3$ \\
\hline
\end{tabular}

glc, glucose; P, plasma; tri, triglycerides; chol, cholesterol; MAP, mean arterial pressure. * $p<0.05$ compared to control; ** $p<0.01$ compared to control; *** $p<0.001$ compared to control

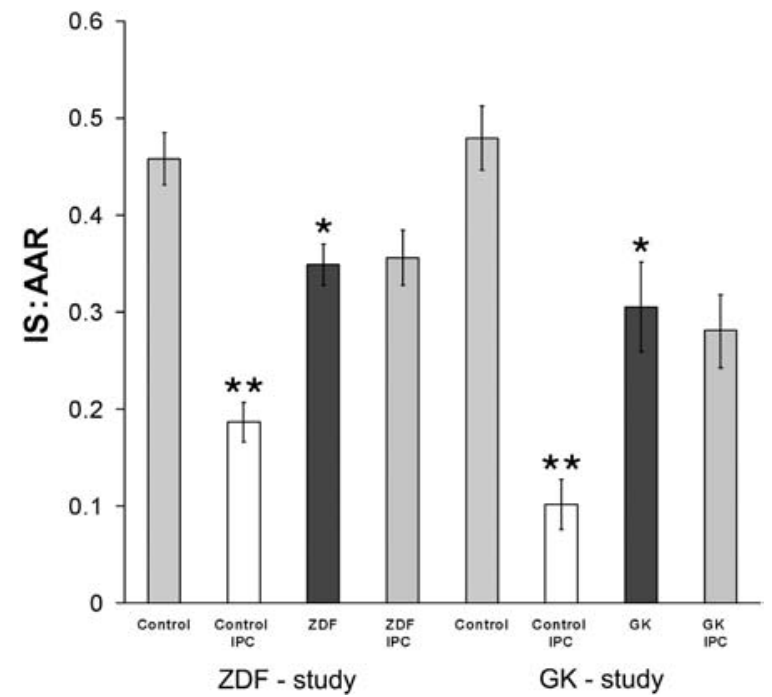

Fig. 1. Infarct size relative to area-at-risk (IS : AAR) in Zucker diabetic fatty (ZDF) and Goto-Kakizaki (GK) rats. Both strains had significantly smaller infarcts than their respective controls. Ischaemic preconditioning (IPC) significantly reduced the IS : AAR in both control groups, but failed to protect hearts from diabetic animals. Data are shown as means \pm SEM. $* p<0.05$ compared to control; ** $p<0.01$ compared to control

elevated NEFA and triglyceride levels compared to control animals, whereas the lean diabetic GK rats had hyperglycaemia, normoinsulinaemia, hypercholesterolaemia and reduced NEFA and triglyceride levels (Table 1). Blood pressure was significantly increased in the diabetic ZDF rats, but not in GK rats when compared to the respective controls (Table 1).

Myocardial infarct size. Ischaemic preconditioning significantly reduced IS : AAR in both control groups $(0.19 \pm 0.02$ vs $0.46 \pm 0.03, p<0.01)$ and $(0.10 \pm 0.03$ vs $0.48 \pm 0.03, p<0.001)$. In contrast IPC failed to protect hearts from $\mathrm{ZDF}(0.36 \pm 0.03$ vs $0.35 \pm 0.02, p=0.84)$ and GK rats $(0.28 \pm 0.04$ vs $0.30 \pm 0.05, p=0.60)$ (Fig. 1). IS : AAR was significantly smaller in the ZDF $(0.35 \pm 0.02$ vs $0.46 \pm 0.03, p<0.05)$ and $\mathrm{GK}(0.30 \pm 0.05$ vs $0.48 \pm 0.03, p<0.01)$ rats than in their respective controls (Fig. 1), but not significantly different between the ZDF and GK groups $(p=0.36)$. AAR : ALV did not differ between groups (ZDF: $p=0.16$, GK: $p=0.52$ ).

Left ventricular function. No difference was found in LVDP or RPP before ischaemia between ZDF or GK groups and their respective controls. IPC improved LVDP during reperfusion in both control groups $(p<0.05)$ (Fig. 2). In contrast IPC failed to improve LVDP in ZDF ( $p=0.40)$ or GK $(p=0.96)$ rats (Fig. 2$)$. There were no differences in LVDP during reperfusion between the ZDF or GK groups and their respective controls. LVDP during reperfusion was significantly lower in ZDF rats than in the GK group $(p<0.05)$.

IPC improved RPP during reperfusion in control animals $(p<0.05)$, but not in diabetic animals (ZDF, $p=0.77$; GK, $p=0.39$ ). RPP during reperfusion was significantly decreased in the ZDF group when compared to their control group $(p<0.05)$, while this was not the case for the GK rats $(p=0.44)$ (Fig. 3). RPP during reperfusion was significantly lower in ZDF than in GK rats $(p<0.05)$.

Coronary flow. There were no differences in coronary flow before or after ischaemia between ZDF and GK groups and their controls. Ischaemic preconditioning did not influence coronary flow during reperfusion in either diabetic or non-diabetic animals (data not shown).

\section{Discussion}

The main new findings of this study are that infarct size after a coronary artery occlusion in both Type 2 diabetes models is smaller than in non-diabetic control animals and that IPC does not protect the heart in obese and lean animal models of Type 2 diabetes. We also observed differences between the two kinds of Type 2 diabetes, since post-ischaemic haemodynamic recovery was significantly smaller in obese diabetic rats than in lean diabetic animals. 




Fig. 2. Left ventricular developed pressure (LVDP) during stabilisation (Stab), regional ischaemia (Isc) and reperfusion. Ischaemic preconditioning (IPC) significantly improved LVDP during reperfusion in non-diabetic animals (full line, filled square) compared to control animals (full line, open square). In

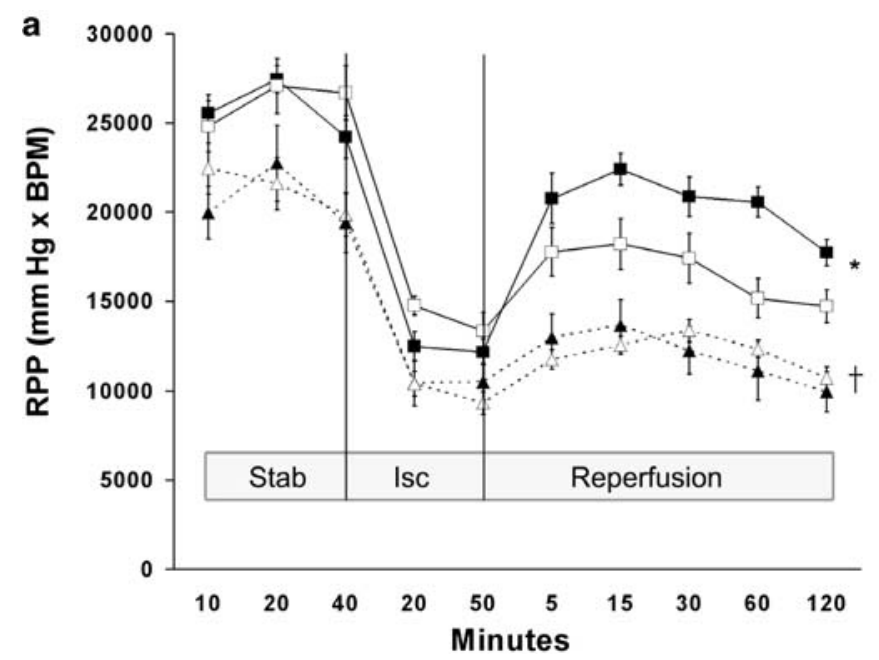

Fig. 3. Rate pressure product (RPP) during stabilisation (Stab), regional ischaemia (Isc) and reperfusion. Ischaemic preconditioning (IPC) significantly improved RPP during reperfusion in non-diabetic animals (full line, filled square) compared to control animals (full line, open square). In contrast IPC had no effect on RPP during reperfusion in Zucker diabetic fatty (ZDF) rats (dashed line, filled triangle) (a) or Goto-Kakizaki (GK) rats (b) (dashed line, filled triangle) compared to control animals (dashed line, open triangle). Data are shown as means \pm SEM. During reperfusion RPP was significantly lower in ZDF rats (a) than in controls. * $p<0.05$ compared to control; $\dagger p<0.05$ compared to $\mathrm{GK}$

Until now studies of myocardial protection and susceptibility to ischaemia-reperfusion in diabetic animals have only been performed in models of Type 1 diabetes. The two models of obese and lean Type 2 diabetes used in the present study $[5,6,7,8]$ were well characterised with respect to circulating blood

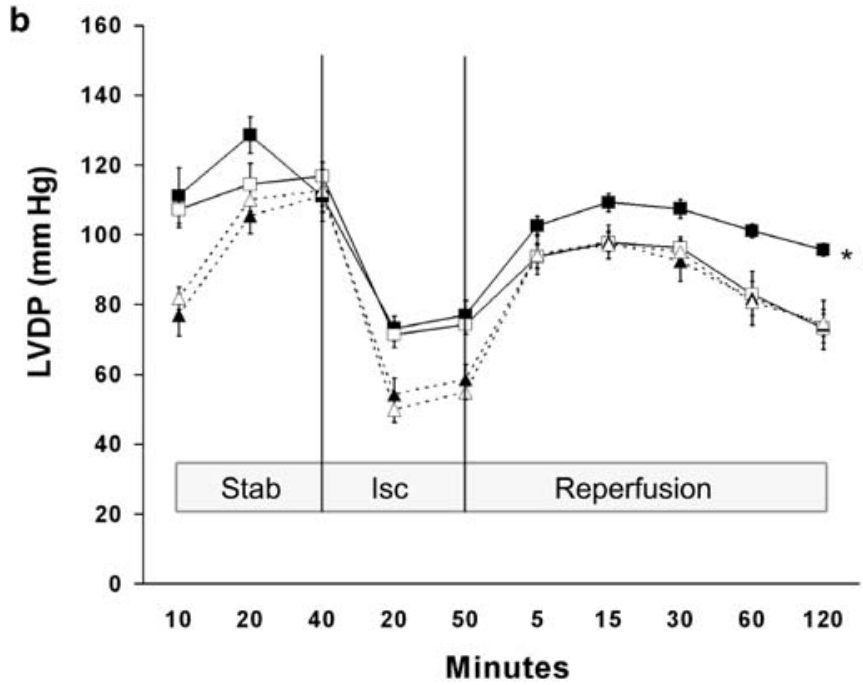

contrast IPC had no effect on LVDP during reperfusion in Zucker diabetic fatty rats (dashed line, filled triangle) (a) or Goto-Kakizaki rats (b) (dashed line, filled triangle) compared to control animals (dashed line, open triangle). Data are shown as means \pm SEM. ${ }^{*} p<0.05$ compared to control animals

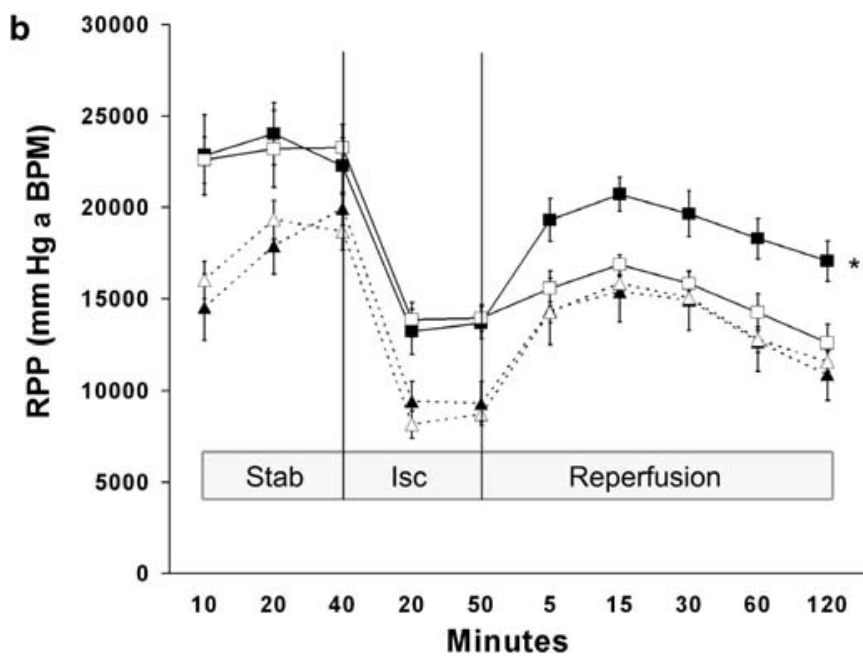

glucose, insulin, triglycerides, cholesterol and NEFA levels. The differences between the metabolic disturbances in obese and lean diabetic rats mimicked the clinical features both of obese Type 2 diabetic human patients with hyperglycaemia, hyperinsulinaemia and hypertriglyceridaemia, and of lean Type 2 diabetic subjects with hyperglycaemia, hyperinsulinaemia and negligible lipid disturbances.

The impact of diabetes on myocardial infarct size after an ischaemic insult in experimental Type 1 diabetes has shown variable results $[9,10,11,12,13]$. The discrepancies can be explained by different experimental conditions. The present study in Type 2 diabetes demonstrates that hearts from diabetic rats are protected against ischaemic damage because infarct size was smaller in both obese and lean diabetic animals than in non-diabetic animals. However, in contrast to a recent study demonstrating increased post-ischaemic left ventricular recovery after low-flow ischaemia [14], our 
data show no improvement of post ischaemic left ventricular function in Type 2 diabetic animals, although infarct size, compared to non-diabetic control animals, was smaller, suggesting a contractile dysfunction in non-infarcted diabetic myocardium. This is supported by a previous study [15], which reported a dissociation between protection against isoproterenol-induced myocardial fibrosis and the haemodynamic response, which was unaffected in a model of Type 1 diabetes.

Animal experiments $[9,16,17]$ and clinical studies in Type 1 and Type 2 diabetes [18, 19] have suggested that the effect of IPC is impaired or even abolished in the diabetic heart, but findings have not been consistent. Discrepancies may be due to confounding factors such as unspecified metabolic and haemodynamic abnormalities and differences in the use of hypoglycaemic agents (i.e. sulphonylureas, metformin and/or insulin). Due to the more variable metabolic disturbances, the effects of Type 2 diabetes on myocardial metabolism appear more complex than those of Type 1 diabetes. Nevertheless, the present results consistently demonstrate that diabetes abolishes the effects of IPC in two different models of Type 2 diabetes. Our data are in accordance with those reported by Ebel and coworkers, who found that late IPC (after 24 hours) in the alloxan model of Type 1 diabetes did not have any cardioprotective effect [20]. The mechanism responsible for the lack of protection afforded by IPC in Type 2 diabetic subjects has yet to be clarified. It has been reported that nitric oxide, protein kinase $\mathrm{C}$ and $\mathrm{K}_{\mathrm{ATP}^{-}}$ channels are involved in IPC in non-diabetic subjects and that nitric oxide metabolism is impaired in diabetic subjects. However, in Type 1 diabetic animals the presence of a functional inducible nitric oxide synthase gene (producing high levels of nitric oxide) seems to aggravate ischaemic damage [21]. Hyperglycaemia is considered a major determinant of the extent of myocardial infarction in experimentally induced Type 1 diabetes and even in the absence of diabetes [22, 23]. Our data demonstrate that hyperglycaemia in itself at the time of ischaemia-reperfusion is not responsible for abolishing the effects of IPC in Type 2 diabetes. In contrast, the persistent hyperglycaemic environment prior to ischaemia-reperfusion may induce a myocardial adaptation which inhibits IPC. The lack of cardiac protection appeared to be quantitatively independent of the degree of hyperglycaemia since no difference in IS : AAR was observed between the two Type 2 diabetes groups, despite a difference in circulating glucose levels. However, the fact that the a priori protection of the diabetic heart is not improved by IPC suggests that common underlying pathways mediate the inherent protection against extension of infarct size afforded by Type 2 diabetes and by IPC in non-diabetic animals, providing further reasons why IPC fails to give protection in Type 2 diabetes.

Even though it is not clear whether findings of different myocardial infarct size in animals with and without diabetes can be extrapolated to humans and have a clinical implication, the loss of the effects of IPC may be one factor responsible for the poor outcomes of diabetic patients with acute myocardial infarction. The present study may explain the finding that the cardioprotective effects of prodromal angina are lost in diabetic patients with myocardial infarction [24].

In conclusion, hearts from both obese and lean Type 2 diabetic animals are protected against myocardial ischaemic injury following a coronary artery occlusion, while leaving post-ischaemic ventricular dysfunction unaffected. Furthermore, IPC does not protect hearts from Type 2 diabetic animals when compared to hearts from non-diabetic animals. The animal models used and the method described here may be a useful tool for studying aspects of the increased cardiovascular morbidity and mortality in Type 2 diabetes.

Acknowledgements. This study was financially supported by the Danish Heart Foundation, the Danish Diabetes Association, Institute of Experimental Clinical Research, Aarhus University and The Novo Nordisk Foundation.

\section{References}

1. King H, Aubert RE, Herman WH (1998) Global burden of diabetes, 1995-2025: prevalence, numerical estimates, and projections. Diabetes Care 21:1414-1431

2. Haffner SM, Lehto S, Ronnemaa T, Pyorala K, Laakso M (1998) Mortality from coronary heart disease in subjects with type 2 diabetes and in nondiabetic subjects with and without prior myocardial infarction. $N$ Engl J Med 339:229-234

3. Zimmet P, Alberti KG, Shaw J (2001) Global and societal implications of the diabetes epidemic. Nature 414:782-787

4. Feuvray D, Lopaschuk GD (1997) Diabetes mellitus and the cardiovascular system. Cardiovasc Res 34:1-2

5. Clark JB, Palmer CJ, Shaw WN (1983) The diabetic Zucker fatty rat. Proc Soc Exp Biol Med 173:68-75

6. Peterson RG, Shaw WN, Neel M-A, Little LA, Eichberg J (1990) Zucker diabetic fatty rats as a model for non-insulin-dependent diabetes mellitus. ILAR News 32:16-19

7. Goto Y, Kakizaki M, Masaki N (1975) Spontaneous diabetes produced by selective breeding of normal Wistar rats. Proc Japan Acad 51:80-85

8. Movassat J, Saulnier C, Portha B (1995) Beta-cell mass depletion precedes the onset of hyperglycaemia in the GK rat, a genetic model of non-insulin-dependent diabetes mellitus. Diabetes Metab 21:365-370

9. Liu Y, Thornton JD, Cohen MV, Downey JM, Schaffer SW (1993) Streptozotocin-induced non-insulin-dependent diabetes protects the heart from infarction. Circulation 88:1273-1278

10. Tani M, Neely JR (1988) Hearts from diabetic rats are more resistant to in vitro ischemia: possible role of altered Ca2+ metabolism. Circ Res 62:931-940

11. Forrat R, Sebbag L, Wiernsperger N, Guidollet J, Renaud S, De Lorgeril M (1993) Acute myocardial infarction in dogs with experimental diabetes. Cardiovasc Res 27:19081912 
12. Feuvray D, Idell-Wenger JA, Neely JR (1979) Effects of ischemia on rat myocardial function and metabolism in diabetes. Circ Res 44:322-329

13. Vogel WM, Apstein CS (1988) Effects of alloxan-induced diabetes on ischemia-reperfusion injury in rabbit hearts. Circ Res 62:975-982

14. Wang P, Chatham JC (2004) Onset of diabetes in Zucker diabetic fatty (ZDF) rats leads to improved recovery of function following ischemia in the isolated perfused heart. Am J Physiol Endocrinol Metab 286:E725-E736

15. Gotzsche O (1982) Lack of cardiotoxic effect of isoproterenol in streptozotocin diabetic rats. A morphometric study of isoproterenol induced fibrosis. Virchows Arch A Pathol Anat Histol 397:83-91

16. Tosaki A, Engelman DT, Engelman RM, Das DK (1996) The evolution of diabetic response to ischemia/reperfusion and preconditioning in isolated working rat hearts. Cardiovasc Res 31:526-536

17. Bouchard JF, Lamontagne D (1998) Protection afforded by preconditioning to the diabetic heart against ischaemic injury. Cardiovasc Res 37:82-90

18. Ghosh S, Standen NB, Galinianes M (2001) Failure to precondition pathological human myocardium. J Am Coll Cardiol 37:711-718
19. Cleveland JC Jr, Meldrum DR, Cain BS, Banerjee A, Harken AH (1997) Oral sulfonylurea hypoglycemic agents prevent ischemic preconditioning in human myocardium. Two paradoxes revisited. Circulation 96:29-32

20. Ebel D, Mullenheim J, Frassdorf J et al. (2003) Effect of acute hyperglycaemia and diabetes mellitus with and without short-term insulin treatment on myocardial ischaemic late preconditioning in the rabbit heart in vivo. Pflugers Arch 446:175-182

21. Marfella R, Di Filippo C, Esposito K et al. (2004) Absence of inducible nitric oxide synthase reduces myocardial damage during ischemia reperfusion in streptozotocin-induced hyperglycemic mice. Diabetes 53:454-462

22. Kersten JR, Schmeling TJ, Orth KG, Pagel PS, Warltier DC (1998) Acute hyperglycemia abolishes ischemic preconditioning in vivo. Am J Physiol 275:H721-H725

23. Kersten JR, Toller WG, Gross ER, Pagel PS, Warltier DC (2000) Diabetes abolishes ischemic preconditioning: role of glucose, insulin, and osmolality. Am J Physiol Heart Circ Physiol 278:H1218-H1224

24. Ishihara M, Inoue I, Kawagoe T et al. (2001) Diabetes mellitus prevents ischemic preconditioning in patients with a first acute anterior wall myocardial infarction. J Am Coll Cardiol 38:1007-1011 